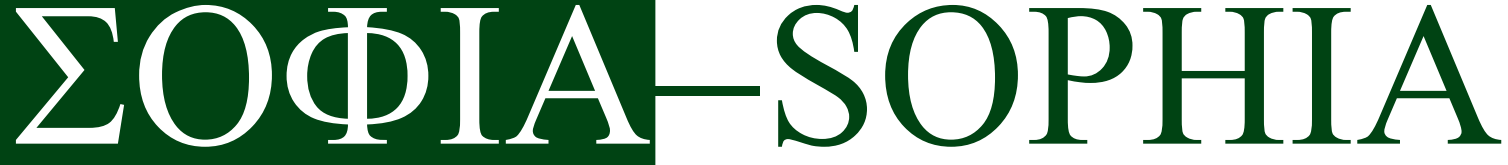

DOI: http://dx.doi.org/ 10.18634/sophiaj.13v.2i.666

\section{Revisión teórica de la discapacidad visual, estudio sobre la importancia de la creatividad en la educación}

\section{Theoretical review of visual impairment, study on the importance of creativity in \\ education}

\section{Revisão teórica da deficiência visual, estudo sobre a importância da criatividade na educação}

Jose Enrique Llamazares de Prado* Ana Rosa Arias Gago** María Antonia Melcon Álvarez***

Información del artículo

Recibido: diciembre de 2016

Revisado: febrero de 2017

Aceptado: Junio de 2017

Cómo citar:

Llamazares, J.E., Arias, A.R., Melcon, M.A. (2017) Revisión teórica de la discapacidad visual, estudio sobre la importancia de la creatividad en la educación. Sophia 13 (2):106-119.
* Facultad de educación. Colaborador del departamento de educación. Universidad de León.

**Dra profesora facultad de educación. Departamento de didáctica. Universidad de León.

***Dra. Profesora departamento de Psicología. Universidad de León. Facultad de educación. Universidad de León.

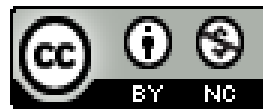




\title{
Resumen
}

En el siguiente trabajo se plantea la importancia en la educación de elementos clave como son la creatividad, y el pensamiento divergente. Esta exposición tiene como principal objetivo la revisión de los estudios de discapacidad visual y creatividad. Pretendemos una reflexión sistematizada de la estimación cuantitativa sintética de todos los estudios disponibles, planteando un marco teórico relacionando la creatividad en la educación del alumnado con deficiencia visual, aportándose valoraciones personales. Dentro de las conclusiones obtenidas, se evidencia la función del potencial creativo por medio del pensamiento divergente en la creación de conceptos e ideas, para la resolución de problemas, con interés no solo en la educación, sino en cualquier ámbito de la vida.

Palabras clave: Creatividad, deficiencia visual, educación inclusiva, estudio bibliográfico, pensamiento divergente.

\begin{abstract}
In the following work, it is raised the importance in education of key elements such as creativity and divergent thinking. This exhibition has as its main objective the review of studies on visual impairment and creativity. We propose a systematized reflection of the synthetic quantitative estimation of all the available studies, proposing a theoretical framework relating creativity in the education of students with visual deficiency, providing personal assessments. Within the conclusions drawn, the role of creative potential is evidenced, through divergent thinking in the creation of concepts and ideas, for problem solving, with interest not only in education but in any field of life.
\end{abstract}

Keywords: Visual deficiency, creativity, bibliographic review, inclusive education, divergent thinking.

\section{Resumo}

Neste trabalho é abordada a importância de elementos chave para a educação, como a criatividade e o pensamento divergente. O principal objetivo desta exposição é a revisão dos estudos de deficiência visual e criatividade. Procuramos uma reflexão sistematizada da estimação quantitativa sintética de todos os estudos disponíveis, formulando um quadro teórico relacionando a criatividade na educação dos estudantes com deficiência visual, fornecendo avaliações pessoais. Nas conclusões obtidas, verifica-se a função do potencial criativo, através do pensamento divergente na criação de conceitos e ideias, para a resolução de problemas, com interesse, não só na educação, mas em qualquer âmbito da vida.

Palavras-chave: Deficiência visual, criatividade, revisão bibliográfica, educação inclusiva, pensamento divergente. 


\section{Introducción}

Podemos afirmar que son escasas las investigaciones (Arnheim, 1990; Tilley, 1991; Mitjáns, 1996, 1997; Connell, 2000; Rodríguez, 2002; Checa, Díaz, y Pallero, 2003; Chinchilla, y Conejo, 2003; Lobato, Martínez y

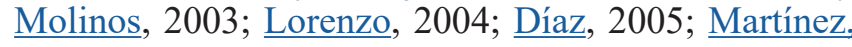
2005a, 2005b; Dosio, 2007, s.f.; Runco, 2014; Toro, 2008; Heredia, 2009; Allen, 2010; Álvarez, 2010; Da Rosa, Goncalves y da Cunha, 2011; Moreno, Huijbregt, y Ramírez, 2012; Espinosa, 2014; Espinosa y Castillo, 2014; Peña, 2014; Aguês Da Cruz, 2016) que abordan la relación entre la ceguera y la creatividad, y menos aún sobre los efectos causados por la privación de la visión en el desarrollo de las capacidades creativas o del pensamiento divergente.

Este escaso interés se debe a la falsa consideración de que las personas con discapacidad visual son incapaces de lograr la interacción en una actividad creativa predominantemente visual, sin interés en las capacidades personales de los sujetos o el desarrollo que pueda mejorar en los mismos o, dicho de otro modo, el arte no implica un desarrollo en el sujeto invidente, ya que al carecer del sentido de la vista se considera poco factible el desarrollo de la capacidad creadora, lo sublime (Torretti, 2008) o la experiencia estética. Hoy en día sabemos que esto no es así, aunque perdura aún esta fundamentación arraigada en algunas personas e instituciones culturales donde poco o nada se da para proporcionar una inclusión factible, aun cuando hay leyes a nivel europeo (Art. 5, 24) ${ }^{1}$ que potencian la inclusión (véase López-Torrijo, 2009).

En este artículo pretendemos una revisión teórica, indagando sobre la importancia en la educación de elementos clave como son la creatividad, y el pensamiento divergente, con el objetivo de revisar los estudios de discapacidad visual y creatividad. Por medio de una reflexión sistematizada de la estimación cuantitativa sintética de todos los estudios disponibles, planteando un marco teórico relacionando la creatividad en la educación del alumnado con diversidad funcional visual, aportándose valoraciones personales.

Resulta imprescindible la educación en la creatividad y en el pensamiento divergente (Álvarez, 2010); aspectos que de inmediato nos hacen pensar en la Expresión Plástica, siendo necesario en todos los niveles de la enseñanza incluir las asignaturas que potencian la creatividad (Romero, 2013). El aprendizaje a través de la creatividad (Marina \& Marina, 2013), se convierte en los niños con diversidad funcional en un "metalenguaje", facilitándose experiencias y progresos educativos.

1. Igualdad y no discriminación (Artículo 5), Educación Inclusiva (Artículo 24). (www.inclusióneurope-org/es/derechos). La ONCE y su Fundación han contribuido en el diseño y defensa de la nueva Estrategia Europea de Discapacidad 2010-2020, que vincula sus propuestas a las Estrategias UE2020, desde la ONCE y su Fundación se aportan estrategias y reivindicaciones a la futura Ley Europea de Accesibilidad

\section{Desarrollo de la creatividad y deficiencia visual}

Para el desarrollo de la creatividad se tienen que dar ciertos elementos específicos: conocimientos precedentes, unos buenos mecanismos de memoria de trabajo, razonamiento y lenguaje apropiado, así como que estos elementos estén en relación con las ideas originales imprescindibles para el germen de conceptos nuevos (Arnheim, 1990; Tilley, 1991; Connell, 2000; Chinchilla, y Conejo, 2003; Lobato et al., 2003; Lorenzo, 2004; Díaz, 2005; Martínez, 2005a, 2005b; Heredia, 2009; Allen, 2010; Limiñana et al. ,2010; Moreno et al., 2012; Espinosa, 2014; Espinosa y Castillo, 2014; Aguês Da Cruz, 2016).

Por lo general, las características mejor valoradas en la creatividad son las planteadas por Guilford (1991; Romero, 2013); la originalidad, la flexibilidad y la fluidez. Otros autores también coinciden con ello (Chacón, 2005; López, 2008; De la Torre, 1981, 1991; Fernández, 2005). No obstante, para algunos investigadores hay un cuarto elemento en relación con el pensamiento divergente que es: la elaboración (Amestoy de Sánchez, 1991; Penagos, 1995, 1997; Romo, 1997; Penagos y Aluni, 2000; Jiménez, Artiles, Rodríguez y García, 2007; Álvarez, 2010). Además, se pueden evidenciar otras variables como son: un contexto adecuado, una alta motivación intrínseca, constancia y capacidad para mantener la propia idea.

En la intervención psicoeducativa de los programas para sujetos con o sin problemas, destacan el entrenamiento de la creatividad cuyo objetivo es desarrollar el pensamiento productivo, la fluidez, la flexibilidad, la resolución de problemas, el autoconcepto, la comunicación y la autopercepción de las propias habilidades personales en el sujeto (Mitjáns, 1997; Garaigordobil y Pérez, 2001; Riley, 2001; Malchiodi, 2003; Rubín, 2009; Limiñana et al., 2010; Conejo y Chinchilla, 2010). Son escasos los estudios que abordan los efectos que puede producirse con la pérdida de visión sobre el desarrollo de las capacidades del pensamiento creativo.

\section{Estudios del factor creativo en personas con discapacidad visual}

Los estudios presentados a continuación, que relacionan las personas invidentes y la creatividad, se podrían englobar en una determinada selección: la correlación de la movilidad y el pensamiento creativo, la inclusión creativa, la imagen creativa, la expresión creativa, el desarrollo de las capacidades y el aprendizaje artístico.

La correlación de la movilidad y el pensamiento creativo (De Bono, 2000) fue planteado por Tisdall, Blackhurst, \& Marks (1971), que destacan un incremento en la movilidad en niños invidentes con una función de 
adecuación a las pautas de riesgo, constituyendo una demostración de pensamiento creativo (Al-Dababneh, al-Masa'deh, y Oliemat, 2015).

Los investigadores Byers-Lang y McCall (1993), abordaron el estudio sobre la inclusión creativa en personas invidentes en la etapa infantil con programas de rehabilitación con base en grupos de pares, concluyendo que un mayor desarrollo de la creatividad en niños invidentes lograría aumentar el grado de expresión corporal, dado que en determinados casos, resulta muy difícil un acercamiento al medio sin la referencia visual (Hodge \& Eccles, 2013).

Respecto a la investigación de Jansson (1988), este autor analizó la imagen creativa comparando sujetos con déficit visual y sujetos normovisuales, utilizando el test "Onomatopeya e Imágenes". Los resultados indicaron que los sujetos con déficit visual poseen altas puntuaciones en la configuración de la imagen creativa en comparación con los normovisuales (Holmes, Hughes \& Jansson, 1998).

La investigación de Lowenfeld y Brittain (1993) estudió la expresión creativa subjetiva del sujeto invidente por medio de la obra escultórica. Para estos autores se distinguían dos modelos diferenciados de creatividad conforme a la estructura mental dominante: el modelo háptico y el modelo visual. Los resultados distinguieron distintos medios de expresión para personas invidentes $\mathrm{y}$ en normovisuales, aunque evidenciaron que resulta equivalente en los dos grupos el proceso creativo. En cuanto a la representación de la imagen corporal, los resultados indicaron que los niños invidentes poseían un grado inferior de desarrollo en la imagen corporal, así como formas menos precisas en comparación con los normovisuales (Witkin, Birhbaum, Lomonaco, Leher, \& Herman, 1968; Millar, 1994; Dulin y Hatwell, 2006; Rubin, 2011; Pinquart y Pfeiffer, 2012; Bregagnolo, 2015).

El desarrollo de las capacidades y el aprendizaje de las habilidades artísticas en sujetos invidentes fueron abordados por diversos autores. La capacidad musical en relación con invidentes y normovisuales, fue planteado por Pitman (1965), influenciada por la teoría de Revesz (1950) y utilizando para el estudio el "Test Wing de Inteligencia Musical". Los resultados indicaron que un alto porcentaje de sujetos invidentes mostraban una mayor capacidad musical en comparación con los normovisuales, sobre todo en los subtets en los que intervenía la percepción auditiva. Esta mayor capacidad musical en los niños invidentes no poseía equivalencia con un elevado potencial, sin embargo, sí poseía correspondencia con un desarrollo más completo de ese potencial (Warren, 1994; Grunwald, 2008).
No obstante, en la investigación con niños invidentes en comparación con normovisuales de Halpin, Halpin \& Torrance (1973), los resultados evidenciaron altas valoraciones en flexibilidad, originalidad y fluidez, en las acciones de pensamiento divergente, concluyendo que los invidentes disponían de una mayor creatividad (Rubin, 2011).

Otro estudio más reciente de la imagen corporal en relación con sujetos invidentes, es el de Pinquart y Pfeiffer (2012), sus resultados demuestran que los adolescentes con discapacidad visual y, en especial, en las mujeres adolescentes, presentaban fuertes asociaciones de imagen corporal con el bienestar psicológico y acoso; en el caso de las mujeres, estas son más susceptibles a la insatisfacción corporal que los hombres adolescentes, cuando no son capaces de satisfacer las normas sociales sobre el cuerpo "perfecto".

Retornando al tema del reconocimiento sensorial, la investigación llevada a cabo sobre la exploración háptica por Kennedy (1980) Vanlierde y Wanet-Defalque (2005) y el posterior estudio de Kennedy y Domander (1981); Warren (1994) evidenciaron que el reconocimiento de los objetos es superior en los invidentes con ceguera adquirida en comparación a los invidentes congénitos.

En conclusión, de los estudios analizados se deriva que obtienen mejores resultados los invidentes respecto a las variables citadas en comparación con los normovisuales. No obstante, poseen nivel similar en el pensamiento divergente y en el proceso creativo, en este caso en la capacidad musical. Por otro lado, obtienen peores resultados los invidentes en la representación de la imagen corporal, que los normovisuales. De tal manera, es aconsejable potenciar aquellos aspectos que impliquen un nivel similar o inferior a los normovisuales, mejorándose la didáctica de las personas con discapacidad visual.

\section{El valor de la educación a través de la creatividad en sujetos invidentes}

Hoy en día hay gran interés por parte de la educación, con especial énfasis en la educación inclusiva que nos compete, en utilizar la inteligencia emocional y la creatividad, como elementos fundamentales para el desarrollo personal, social, para fomentar una mayor implicación de los individuos en la sociedad. Respecto a: "La educación inclusiva ha de ser una respuesta educativa dada a las personas con diversidad funcional" (Lozano, Cerezo y Alcaraz, 2015:21).

Dentro del contexto educativo, la creatividad (Romo, 2012) se establece como un proceso de actividades consideradas artísticas, pero generalmente, sin contemplar los procedimientos creativo-divergentes. 
Por ende, la creatividad es: "Un modo personal y original de pensar, sentir y expresarse que se aparta de los modelos socioculturales vigentes o circundantes y da como resultado trabajos distintos, a menudo, originales y valiosos, en los distintos sujetos" (De Prado, 1988, p. 20; Fernández, s.f.).

El uso de la creatividad como elemento para mejorar la calidad de la educación de sujetos con necesidades educativas especiales no es un tema reciente, los predecesores en este tipo de investigación fueron Houtzy Phillips (1976), Khatena (1976) y Uno, Gargiulo, Sears, Mauter \& Rowe (1976); Duarte (2003), analizando los aportes de los estudios sobre la creatividad, así como su incorporación a los planes docentes de los currículos escolares, en concreto en materias de educación para personas con discapacidad, destacando la premisa de que no se mostraron distinciones destacadas en las dimensiones de creatividad. Arnáiz (2003) afirma:

\begin{abstract}
La existencia de un currículum en el que el alumnado con necesidad específica de apoyo educativo participe al máximo de sus posibilidades es fundamental si queremos que las escuelas sean para todos. Se hace, por tanto, necesario un currículum flexible, abierto y con carácter interdisciplinar para que todo el alumnado pueda aprender junto, adoptando una estructura de aprendizaje cooperativo en la que todos se vean estimulados a cooperar, a ayudarse unos a otros, a aprender más y mejor ( $\mathrm{p}$. 25).
\end{abstract}

Pudiéndose dar una adaptación más individualizada cuando se considere necesario, y no desarrollar programas paralelos al ordinario enfocados hacia un tipo de alumnado especifico (Díaz, 2009).En el ámbito didáctico, la inclusión de métodos, actividades, técnicas creativas y artísticas en el plan docente constituye la intención de lograr una enseñanza para el éxito (Garrido, 1988; Fernández, s.f.).

En el ámbito de los estudiantes invidentes, a causa de la disminución de la entrada sensorial, el aprendizaje se da como resultado de la acción reciproca táctil-kinestésica y el sentido auditivo. La cantidad de información que el sujeto invidente obtiene del entorno es recogida de modo fragmentario, a diferencia de los normovisuales. De ello se deriva la importancia en el uso de estos canales de comunicación en la didáctica, destacando el uso de todos los sentidos en la enseñanza para fomentar una educación multisensorial (Dosio, s.f.). En el alumnado con discapacidad visual, la actuación del docente en materia creativa favorece la adaptación a las posibilidades reales para cada alumnado, motivándoles a elaborar conceptos y desarrollar actividades que tengan su base en los conocimientos y las expresiones (Fernández, s.f.).
Para Runco (2014) hay distintas razones para plantear con entusiasmo el potencial creativo en el alumnado con necesidades educativas especiales: por un lado, este aparentemente está considerablemente diversificado, así como la importancia que posee la motivación en las actividades creativas.

Para Novaes (1979) es primordial apoyar la necesidad del desarrollo creativo fomentando un ambiente favorable para la expresión y la generación de ideas, e innovaciones, destacando su aplicabilidad en las aulas y con énfasis en el inicio en edades tempranas continuando a lo largo de su formación (Frías, 2013), posibilitando con ello la mejora en otras áreas temáticas escolares. Cabe exponer que todas las personas poseemos en mayor o menor grado potencial creador capaz de ser desarrollado. La diferencia a la hora de manifestarse la creatividad entre los diferentes sujetos se debe a la herencia y a la educación.

La investigación de Sternberg y Lubart (1997) respecto a la capacidad creadora ha evidenciado que determinados sujetos disponen de altas competencias en un cierto ámbito de desempeño o en algunos, con lo que resulta factible estar dotado de capacidad creadora en cierto aspecto y no estarlo en otro. En consecuencia, estos autores afirman que segregar a los sujetos menos creativos de los más creativos, no tiene sentido. Siendo igualmente aplicable a cualquier tipo de alumnado con o sin discapacidad.

Siguiendo a Carpio (1999) un sujeto será creativo únicamente en aquellos ambientes en los que haya alcanzado una configuración adecuada de las competencias a la resolución de problemas. Es en la educación donde se desarrollan unas competencias básicas, no obstante, se necesita de un planteamiento de circunstancias que posibiliten una configuración diversa y no exclusivamente de una única solución.

En el libro Atmósferas creativas. Juega, piensa y crea, Betancourt y Valadéz (2005) especifican que un ambiente creativo, es aquel que fomenta un estado adecuado de activación tanto afectiva como cognitivamente y facilita en la realización de tareas grupales el rendimiento productivo.

Las ventajas de dotar al alumnado de un ambiente creativo en el caso que nos ocupa, la discapacidad visual, con la eliminación de barreras para poder dotarles de libertad sin condicionamientos externos, son diversas, como son: mejora de su autoestima, su autoconcepto, su motivación y capacidad expresiva, se siente más seguro y desarrollan una mejor comunicación y generación de ideas, así como una mejora evidente en la forma de expresarlas. No obstante, la mayoría de los estudios sugieren la necesidad de indagar más para obtener 
resultados sólidos en las investigaciones (Halder, y Datta, 2012; Mishra, y Singh, 2012; Datta, 2014, 2015).

De las aportaciones expuestas en la obra de Betancourt y Valadéz (2005), se extrae la necesidad de que el estudiante pueda aprovechar las capacidades personales con posterioridad, cambiar el papel del profesor como protagonista por un rol más accesible, regulando las tareas de una manera más cercana, favorecer la implicación del alumnado sin exclusión, aunque no forzando a los que no quieran, por timidez o vergüenza, además de favorecer el interés de los trabajos para que sean atractivos al alumnado. Coinciden estas aportaciones con las planteadas en el Modelo para la Estimulación del Pensamiento Creativo. El MEPC valora la creatividad como un elemento del pensamiento, así como una competencia de cualquier persona en mayor o menor grado (Duarte, 2004).

Podemos destacar a Marín (1995) como el investigador principal que postuló la defensa de la creatividad como pilar fundamental en la acción educativa; hipótesis que tiene más fuerza en una época de cambios como la que vivimos, donde la competitividad y el desarrollo tienen que ser lo mejores posibles. Por lo tanto, incentivar en la enseñanza el pensamiento divergente es un recurso principal para el progreso de cualquier centro educativo, para formar un alumnado con más capacidad de resolución, innovación y, salidas a las limitaciones que se les planteen a lo largo de su desarrollo.

Es evidente que la mejora curricular de los centros siempre es posible, y que tienen que estar abiertos a mejoras y cambios que proporcionen un mejor desarrollo para su alumnado, por tanto, es la creatividad; como defiende De Prado (2003), un factor educativo que posibilita que el alumnado y el profesorado reformulen ideas, e imágenes, reconstruyendo estructuras mentales, para obtener un mejor razonamiento por medio de la elaboración de opiniones e ideas diversas, relaciona experiencias personales, para configurar la creación de ideas, así como proyectarlas en la práctica, resolviendo obstáculos.

En la rama de la práctica pedagógica, la terminología de la creatividad se entiende como el progreso de las capacidades para tener unas conclusiones nuevas e innovadoras, que derriben obstáculos presentados en el aula. Respecto a la definición de la creatividad en el campo educativo De Prado (2003), la menciona como el potencial humano formado por elementos cognitivos, intelectuales y afectivos, por medio de un ambiente creativo se percibe con claridad para producir nuevos materiales, con un enorme valor social y transmitirlos dentro del contexto social. Integrando en este término las propiedades elementales de la creatividad como son: el sujeto, el proceso, el producto y el medio (Garaigordobil y Torres, 1996; Monreal, 2000; Penagos y Aluni, 2000).
Por otro lado, la definición que aplica Penagos y Aluni (2000) la delimita como la generación de elementos y/o comportamientos destacados para un conocimiento escaso o un hecho que requiera habilidad. Entendiéndose desde la idea de producción cuando la valoración de la creatividad se le otorga un valor destacado a la habilidad que conllevara el resultado o producto final de la idea.

Por su parte, Mendoza, (2001) y Rosa, (2008), mantienen la teoría de González y Mitjáns (1999) afirmando: "La creatividad es un proceso de descubrimiento o producción de algo nuevo, que cumple determinadas exigencias sociales y en el cual se da el vínculo de los aspectos cognitivos y afectivos de la personalidad" (p.24). En conclusión, encontrar o descubrir la novedad donde hay unas pautas sociales de exigencia que delimitan que algo pueda ser considerado creativo, así como la importancia de las emociones y la personalidad de la artista plasmada en la configuración de la idea creativa.

Aún reconociendo que la creatividad es un elemento con diversos significados es apropiado comprender ciertas propiedades de los procesos creativos (De la Torre y Marín, 2003); estas pueden resumirse del siguiente modo: el acto creativo es intrínsecamente exclusivo del hombre, el sujeto por medio de sus acciones refleja ideas, sentimientos, emociones, de modo equivalente esta acción es deliberada, dirigida, con un propósito, obtener soluciones respecto a las limitaciones y obstáculos, exteriorizar ideas o ejecutar un pensamiento. El hombre se distingue por inferir la premeditación en los hechos de más relevancia. Lo que nos lleva a deducir que la transferencia de la información en la ejecución y la formación de nuevas ideas juega un papel crucial en la creatividad, sin transmisión quedan sin el elemento principal de la comunicación, la creatividad como tal es más que el factor de inspiración se tiene que reconfigurar el entorno o aquello que nos rodea por medio de la mente del hombre, y poder trasladar estas ideas a través del acto creativo, cambiando las señales por signos, con una fuerte carga de originalidad y novedad, de tal manera es escrita por los investigadores (Tisdall, Blackhurst \& Marks, 1971; Halpin, Halpin \& Torrance, 1973; Rubin, 2011; Giúdice, 2013; Al-Dababneh, al-Masa'deh, y Oliemat, 2015) como la competencia para obtener conclusiones de pensamiento innovador y singular valorando estos elementos determinantes del pensamiento divergente.

En la creatividad juega un papel importante la emoción, y los procesos emocionales, por ende, actualmente se trata de impulsar su aplicabilidad en las aulas para mejorar los currículos escolares a través de la gestión emocional, fomentando la empatía pilar elemental si queremos escuelas innovadoras. En este sentido, cabe citar como ejemplo los centros "Change Maker": Escuela Sadako, y Amara Berri, decidiendo el alumnado 
qué desea aprender incrementando exponencialmente la motivación e interés por lo que aprende y además se le enseña al alumnado a utilizar su propio lenguaje para compartirlo con los demás (véase Pérez-Pereira \& Conti-Ramsden, 2013). Hoy en día, juega un papel relevante la teoría de Gardner (2011), las inteligencias múltiples, la educación emocional y la tecnología para el desarrollo de una mejora educativa, en la formación de las escuelas innovadoras.

La actividad creativa posee una condición cambiante, el sujeto creativo valora el entorno, le influye, lo reestructura, adaptándolo según sus criterios, consiguiendo un cambio innovador. En el campo didáctico, juega un papel vital la acción del profesorado, (Nuñez, 2001; Rodríguez, 2003; Caballo y Núñez, 2013) en tanto a la adecuación, como la relación de los contenidos de los currículos escolares respecto a los grupos de estudiantes que dispongan, valorando los intereses y las virtudes, así como las necesidades de cada alumnado. Se les debe dotar de autonomía creativa, sin bloqueos creativos (Muñoz, 2015), en un entorno que dote de sentimiento de seguridad para expresar cualquier idea o emoción sin temor al ridículo, en contraposición del alumnado donde predomina el modelo estándar de ejecución, dado que no son capaces de crear o innovar fuera de la norma.

El progreso de cada sujeto que desarrolla una actividad artística está vinculado a las experiencias personales, en consecuencia se tiene que tratar desde una perspectiva individual, y no guarda relación con la edad. Además, lo importante es el proceso creativo y no el resultado material. Por lo tanto, dar pautas estrictas para un modelo concreto de población no resulta factible, a pesar de ello, todos los estudios (Checa et al, 2003; Tapia, 2007; MEC, 2012) evidencian que el desarrollo del alumnado invidente es igual al de los normovisuales, en ciertos casos, este desarrollo puede ser más sosegado, no obstante, hay que evidenciar que hasta el periodo de los trece años es cuando los parámetros de desarrollo quedan igualados. Por otro lado, ciertos sujetos con discapacidad en mayor medida pueden desarrollar habilidades creativas en comparación a sujetos sin discapacidad ( $\underline{\text { Rocío }}, 2011)$.

Podemos finalizar este apartado diciendo:

“Todos los niños tienen derecho a la educación y a todos se les debe dar la oportunidad de alcanzar y mantener su máximo potencial en términos de capacidad cognitiva, emocional y creativa, aprendiendo, siempre que sea posible, juntos, independientemente de las características, intereses, capacidad y necesidades de aprendizaje de cada alumno" (Pujolás, 2010).

\section{Conclusiones}

La educación es un pilar básico de cualquier sociedad, en consecuencia, ha de ser accesible para todos, a través de esta se puede potenciar la creatividad (Giúdice, 2013) y con ello, el pensamiento divergente, facilitando la formación de nuevas ideas y conceptos, así como nuevas vías de resolución de problemas, con interés no únicamente en la aplicabilidad escolar, sino para cualquier ámbito de la vida en general.

El uso de la creatividad no solo implica beneficios en el alumnado, también en el propio profesorado (véase Bae, Song \& Kim, 2012) a través de una formación continuada, posibilitando el desarrollo de la inteligencia creativa con un mayor desarrollo del pensamiento divergente, y un mayor contacto con el alumnado con ejercicios que impliquen la participación activa del alumnado y del profesorado a través de ejercicios cooperativos como el brain storming, brain writing, seis sombreros para pensar, método 635 , etc... Las posibilidades en el profesorado implican una mejor predisposición a la formación de nuevas ideas, una mentalidad más abierta, planteamientos innovadores, propuestas de mejora de la adaptación de los currículos escolares posibilitando una mejora de la educación.

Analizados los siguientes estudios es de vital importancia la aplicabilidad creativa en el contexto escolar, cabe citar los trabajos de Kirst (2010) y Da Rosa Goncalves y da Cunha (2011), de naturaleza cualitativa, se realizó en el caso del trabajo realizado por Kirst (2010) una experiencia taller de Arte Contemporáneo para públicos ciegos y videntes. Con la creación de material didáctico, los dos tipos de alumnados interactuaron con los materiales que suscitaban un diálogo colectivo con el arte. En el análisis de los datos se observó que los invidentes pueden conocer el arte contemporáneo por medio de aprendizajes multisensoriales, así como en las obras que necesiten de poca o ninguna adaptación; pudiendo ser aplicable en las aulas tanto en la enseñanza formal y no formal, utilizando enfoques que sean aplicables tanto a los deficientes visuales como a los normovisuales.

En otro estudio de vertiente didáctica creativa en personas con discapacidad visual es el tratado por Ruiz (2004), donde se establece un programa de innovación docente desde el ámbito escolar y museístico, integrado dentro de un proyecto de innovación docente de la Universidad de Sevilla, instruyéndose al alumnado universitarios acerca del modo más eficaz de orientar y ayudar a una persona invidente en la captación de obras de arte. 
Las actividades artísticas con niños invidentes y con déficit visual en talleres corroboran que tienen un desarrollo creativo que los conecta con los instructores de ese taller, proporcionando un desarrollo en la personalidad de cada alumnado. Estas acciones en talleres proporcionan circunstancias semejantes a las del juego, dotándoles de una mejor expresividad para manifestar sus ideas, pensamientos, sea por la narración o por la creación escrita o dibujada. Facilitando la comprensión de distintas artes se fomenta una mejora en distintas vías de expresión. Cabe citar a dos artistas de USA: R. Nachum que aúna la poética con el Braille en sus óleos y L. Brozgol el cual enseña cerámica a invidentes.

Hay que decir que en la historia del arte hay ejemplos de artistas: Cassatt, Degas, Monet, Munh, O'Keeffe, Pissarro, que sufrieron en algún momento de su vida alguna discapacidad visual y esto no les impidió desarrollarse y mejorar como artistas, incluso mejoraron la creación artística de su momento, este estudio queda reflejado en la investigación de Arqué (2005). En la cual se evidencia los preconceptos tópicos de las limitaciones sensoriales, las cuales no restan validez e importancia a sus propuestas estéticas. No obstante, se han desarrollado propuestas para una mejora en la inclusión cultural que acercan estos planteamientos en U.S.A. como el Moma o el Metropolitan. En España cabe citar la experiencia didáctica en el Museo de Cádiz reflejada por García (1989) en Cuadernos del Sureste (Moreno et al., 2012).

Tenemos que comprender que el arte es una herramienta educativa tan válida como otras, siendo además un instrumento de mejora y crecimiento personal, así como un medio de integración para los distintos ámbitos educativos. El uso del arte en la educación favorece, el desarrollo de las habilidades personales, la motivación, la confianza en sí mismo, la autoconciencia, la generación de ideas facilitando la resolución de problemas, se favorece un trabajo más desarrollado donde el factor de ansiedad no es un obstáculo, así como el aumento de la autoestima personal por los logros o hechos obtenidos o viendo lo que uno mismo puede desarrollar. Siendo estos aspectos importantísimos en la aplicabilidad del aula para cualquier disciplina escolar, el potenciar la creatividad ayuda a la gestión y la toma de decisión de las diversas asignaturas así como la resolución de las mismas.

Como propuesta de mejora, dentro de las escuelas y centros culturales se tendría que potenciar y fomentar el desarrollo del contacto creativo y estético a través del contacto directo, así como la vinculación in situ con artistas y con profesionales mientras realizan sus composiciones, facilitando el acceso a grupos de personas con discapacidad visual para desarrollar el potencial creativo y poder experimentar y aprender de primera mano del contacto creativo, pudiendo desarrollar la capacidad de plasmar sus ideas, pensamientos y sentimientos en la obra de arte. Además, el factor de sensibilización del colectivo normovisual jugaría un papel importante pudiéndose facilitar recorridos culturales con inhibición sensorial (una mirada invisible de lo que nos rodea) aprendiendo a reconocer las formas a través del sistema háptico, así como los olores o el sonido, lográndose una mayor empatía y concienciación de la gente que en ocasiones no valora que en nuestra sociedad hay personas con diversidad funcional en este caso visual.

Para futuras líneas de investigación consideramos que en materia de potenciación de la creatividad se lograría un desarrollo a través de las actividades, tales como: la ejercitación de la memoria, la sinestesia, la programación neurolingüística, la acción teatral y la danza.

Poder evidenciar los beneficios que implica la ejercitación de la memoria sobre todo en la atención temprana en el alumnado con discapacidad, bajo una cooperación de la escuela y el hogar, facilitando el progreso educativo de este tipo de alumnado bajo un discurso educativo unificado y común, para posteriormente en cursos superiores poder trabajar con la asociación de la memoria y el arte, por medio de ejercicios prácticos, destacando en la actividad creativa en el proceso creativo el uso de la memoria y la imaginación, para trasladar desde la percepción háptica y del recuerdo, la experiencia adquirida por la persona con discapacidad visual, así como sus sentimientos y emociones, por medio de la composición de obras artísticas.

En segundo lugar, desarrollar las implicaciones que supone la sinestesia o fusión de los sentidos ( $\underline{\text { Salas, }}$ 2015), descrita como un conjunto de estados cognitivos en relación a la unión de los sentidos, o dicho de otro modo, entendida como el arte de ver la música, oler los colores o tocar las emociones, nada estudiada en las personas con discapacidad. Es una hiperactividad de distintas áreas del cerebro, es una mutación de carácter genético pero también se puede dotar al sujeto con herramientas culturales adecuadas, la investigadora García (2008) desarrollo una asignatura en materia sinestésica para el conservatorio de la ciudad de Sevilla, con una base teórica y otra practica con la unión de la música y de diversos actos escénicos, para el campo artístico cabe exponer: "La riqueza de modalidades de sentidos cruzadas que presenta la sinestesia, es una metáfora de la asociación de ideas y técnicas de disciplinas cruzadas que impulsan tanto la metodología neurológica como el Arte" (García, 2008: 5). 
Además, actualmente disponemos de la posibilidad del uso del arte digital y emplear elementos tecnológicos, para poder desarrollar este tipo de arte sinestésico (Hertz, 2006), por medio de máquinas que relacionan nuestros sentidos (Layden, Söffing \& Schmidtke, 2015), para una particular interpretación de lo que nos rodea, pudiendo ser usado para el aumento de la percepción, el análisis o el modo de vincular o recordar elementos ligados a otros componentes como sensaciones o emociones que nos generan en el momento de percibirlos. Así pues, se puede dar la creación de materiales específicos en la apuesta del arte contemporáneo multisensorial o la cooperación del propio alumnado en la creación de este material para su deleite posterior.

Por otro lado, dentro de la corriente de la programación neurolingüística, método al servicio de la inteligencia emocional y la gestión emocional, cabe destacar la distinción de que cada persona posee un canal sensitivo para entender lo que le rodea y relacionarse con las personas que le rodean, siendo algunas personas más visuales, otras más auditivas y un tercer grupo más táctiles (Romo, et al., 2006). Dependiendo del nivel de desarrollo si predomina en uno de los anteriores nos determinara el modo en que aprendemos y adquirimos información de lo que nos rodea y la relación de nuestras preferencias y gustos personales, de tal manera que, juegan un papel destacado dependiendo de la personalidad. Por ello, consideramos que poder abordar un estudio comparativo sobre estas características con sujetos con discapacidad visual y sin discapacidad, aportaría información útil para el estado de la ciencia y podría servir de apoyo para la gestión emocional en las aulas a través de la programación neurolingüística.

Por otra parte, con el uso de modelos intergeneracionales con la mejora cognitiva, puede ser de gran utilidad para el estado de la ciencia poder medir las diferencias que supone la implementación artística desde el punto de vista del empleo de baterías psicológicas. Poder dar un aprendizaje que pueda agrupar varias generaciones, con actividades teatrales desarrolladas para potenciar la creatividad, la improvisación y beneficiado por la propia experiencia de las personas mayores.

En el caso del alumnado con discapacidad visual, con ceguera y baja visión, juega especial relevancia poder potenciar la expresividad corporal, en representaciones que logren unir el baile junto con la realización de talleres teatrales, hay ejemplos como: Perú, bajo la dirección de la Dra. Llanos Zuloaga (véase, Llanos, 2006) en el desarrollo de la "Danza de la vida o biodanza", para mujeres invidentes especialmente, pero aún no se ha enlazado con representaciones teatrales y las oportunidades que representa para la exposición de obras representadas tanto con miembros con discapacidad como sin ella, logrando así una autentica inclusión artística.
En otros países, como México, en Puebla, bajo el título "el otro cielo, danza desde la ceguera" es un espectáculo inspirado en dos poemas de Mario Benedetti: No te rindas y Oro cielo, acompañados con música de Chopin, Ryuchi Sakamoto, Rene Aubri y Claude Debussy, bajo la dirección de la psicóloga, Lorena Nieva Bernal se apuesta por la participación intergeneracional con 41 bailarines con edades comprendidas entre $\operatorname{los} 8$ a 84 años.

Cabe exponer, en materia de cooperación internacional inclusiva, en México, Puebla "My Dream”, un grupo de Artistas con Discapacidad de China (GADC), que combina el arte en sus diferentes modalidades, conjuntando el talento de más de 40 artistas con discapacidad. Poder desarrollan movimientos de intercambio cultural entre países, con profesionales con discapacidad es un paso que tenemos que tener en cuenta, para poder dotar a la sociedad de los avances inclusivos que se merece y enriquecernos de las posibilidades que nos brinda está posibilidad cultural.

\section{Referencias bibliográficas}

Arnáiz, P. (2003). Educación inclusiva. Una escuela para todos. Málaga: Aljibe.

Arnheim, R. (1990). Aspectos perceptuales del arte para ciegos. Journal of Aesthetic Education 24, 3.

Aguês Da Cruz, S. D. (2016). Do paradigma do ver ao do tocar. $O$ devir háptico na criaçao artistica contemporanea. Tesis doctoral no publicada. Universitat Politècnica de València. doi:10.4995/ Thesis/10251/61441.

Al-Dababneh, K., al-Masa'deh, M. y Oliemat, E. (2015). The effect of a training programme in creativity on developing the creative abilities among children with visual impairment. Early Child Development and Care, 185(2), 317-339.

Allen, P. (2010). Arte-terapia: guía de autodescubrimiento a través del arte y la creatividad. Madrid: Gaia.

Amestoy de Sánchez, M. (1991). Desarrollo de habilidades de pensamiento: Creatividad. Guía del instructor. ( $1^{\text {a }}$ Ed.). (UAN). México, D. F.: Trillas.

Arqué, L. (2005). Arte Ceguera. Integración, 45, 17-24. Once.

Álvarez, E. (2010). Creatividad y Pensamiento Divergente. Desafio de la mente o desafio del ambiente. Interac. Recuperado de: http://www. academia.edu/7684124/CREATIVIDAD Y PEN S A M IEN T O D I VERGEN TE Desaf\%C 3\%ADo de la mente_o desaf $\%$ C $3 \%$ ADo del ambiente. 
Bae, S., Song, J. \& Kim, H. (2012). Teachers' creativity in career technical education: The mediating effect of knowledge creation practices in the learning organization. The Korean Social Science Journal, 39(1), 59-81.

Betancourt, J. y Valadéz, M. (2005). Atmósferas creativas. Juega, piensa y crea. Ciudad de México: Manual Moderno.

Bregagnolo, E. N. (2015). Taller "la educación inicial" (Doctoral dissertation, Universidad Nacional del Nordeste). Recuperado de http:// hum.unne.edu.ar/academica/ambientacion/ modulos/modulo inicial.pdf.

Byers-lang, R. E. \& McCall, R. A. (1993). Peer support groups: Rehabilitation in action. Review: Rehabilitation and Education for Blindness and Visual Impairment, Vol. 25(1), 32-36.

Caballo, C. y Núñez, M. (2013). Personas con discapacidad visual. En Verdugo, M.A. \& Schalock, R.L.Discapacidad e inclusión: manual de docencia. Salamanca: Amaru Ediciones.

Carpio, C. (1999). La creatividad como conducta. En Bazán, A. (comp) Aportes conceptuales y metodológicos en Psicología Aplicada. Ciudad de México: Instituto Tecnológico de guadalajara.

Connell, J. (2000), Aesthetic experiences in the school curriculum, Assessing the value of Rosenblatt's transactional theory, Journal of Aesthetic Education, 34, (1), 27-35.

Conejo, I. y Chinchilla, M. (2010). ¿Puede la terapia artística servir a la educación? EA, Escuela abierta: revista de Investigación Educativa, (13), 69-96.

Chacón, Y. (2005). Una revisión crítica del concepto de creatividad. Revista actualidades investigativas en educación, 5(1). pp. 1-30. DOI: http://dx.doi. org/10.15517/aie.v5i1.9120.

Checa, J., Díaz, P. y Pallero, R. (2003). Psicología y Ceguera. Manual para la intervención psicológica en el ajuste a la deficiencia visual. Madrid: Once.

Chinchilla, M. y Conejo, I. (2003). Creatividad, expresión y arte: Terapia para una educación del siglo XXI. Un recurso para la integración. EA, Escuela abierta: revista de Investigación Educativa, (6), 129-148.

Da Rosa, M., Goncalves, I. y da Cunha, S. (2011). El Profesor de Arte para Niños Ciegos: Prácticas Pedagógicas en Dos Realidades. Brasil: UDESC. Recuperado de: http://www.saece.org.ar/docs/ congreso4/trab24.pdf.
Datta, P. (2014). Self-concept and vision impairment: A review. British Journal of Visual Impairment, 32(3), 200-210.

Datta, P (2015). Autoconcepto y discapacidad visual: una revisión bibliográfica. Madrid: Once.

De Bono, E. (2000). Pensamiento creativo: El poder del Pensamiento Lateral. Manual de creatividad. Argentina: Editorial Paidós Plural.

De Prado, D. (1988). Técnicas creativas y lenguaje total. Madrid: Narcea.

De Prado, D . (2003). La creatividad, motor de la renovación esencial de la educación. Educrea Universidad de Santiago de Compostela: Lubrican.

De la Torre, S. (1981). Creatividad: Qué es, cómo medirla, cómo potenciarla. Barcelona: Sertesa.

De la Torre, S (1991). Evaluación de la creatividad. Madrid: Escuela Española.

De la Torre, S. y Marín, R. (2003). Manual de la Creatividad. Barcelona: Ediciones Vicens Vives.

Díaz, A. M. (2005). Metodología para la educación artística en niños ciegos y baja visión. Santiago, Chile: Escuela de Niños Ciegos Santa Lucía Recuperado de http://www.santalucia.cl/ archivos/ponencia arte ceguera.doc.

Díaz, M. (2009). El Alumnado con Deficiencia Visual. Necesidades y respuesta educativa. Innovación y Experiencias Educativas. (14).1-8.

Dosio, P. (2007). Apuntes sobre el arte de los no videntes. Folleto Vicerrectoría Académica, Biblioteca Mario Carvajal, Área Cultural, Universidad del Valle: Colombia. Recuperado de http://www.minedu.gob.pe/webdipecud/ descarga/Apuntes $\% 20$ sobre $\% 20$ el $\% 20$ arte $\% 20$ de\%201os\%20no\%20videntes.doc.

Dosio, P. (0000.). La educación y los no videntes. Recuperado de http://www.pasoapaso.com.ve/ index.php/unadecadaretratando/item $/ 480 \mathrm{La} \% 20$ educaci $\% \mathrm{C} 3 \% \mathrm{~B} 3 \mathrm{n} \% 20 \mathrm{y} \% 201 \mathrm{os} \% 20 \mathrm{no} \% 20$ videntes.

Dulin, D. y Hatwell, Y. (2006). The effects of visual experience and training in raised-line materials on the mental spatial imagery of blind persons. Journal of Visual Impairment \& Blindness (JVIB), 100, (07) 414-424.

Duarte, E. (2003). Creatividad como un recurso psicológico para niños con necesidades educativas especiales. Sapiens: Revista Universitaria de Investigación, 4(2), 13-32. 
Duarte, E (2004). Educación, aprendizaje y cognición. Teoría en la práctica. En S. Castañeda (Coord.) Modelo para la Estimulación del Pensamiento Creativo. Ciudad de México: Manual Moderno. pp.501-514. Recuperado de http://www.psicologia.uady.mx/documentos/ publicaciones libros/MODELO PARA LAESTIMULACION.pdf.

Espinosa, R. (2014). El proceso creativo de personas invidentes como una forma de expresión comunicativa. Creatividad y sociedad: revista de la Asociación para la Creatividad, (22), 6-34.

Espinosa, R. y Castillo, H. (2014). Análisis y evaluación de la generación de iconos mentales en personas invidentes a partir de la percepción virtual táctil utilizando realidad virtual y sistemas hápticos. Icono14, 12(2), 295-317.

Fernández, E. R. (0000). La Creatividad en la educación de alumnos con necesidades educativas especiales. Recuperado de http://www.iacat. com/revista/recrearte/recrearte01/elena.htm.

Fernández, E. (2005). La creatividad en el desarrollo de los niños ciegos. Universidad de Santiago de Compostela: Creación integral.

Frías, P. (2013). Encrea: docentes con entrenador personal en creatividad Estudio de caso único con línea base múltiple. Creatividad y sociedad: revista de la Asociación para la Creatividad, (21), 4-27.

Gardner, H. (2011). Las inteligencias múltiples: La Teoría en la práctica. Barcelona: Paidós.

Garaigordobil, M. y Torres, E. (1996). Evaluación de la creatividad con sus correlatos con inteligencia y rendimiento académico. Revista de Psicología, 18 (1), 87-98.

Garaigordobil, M., y Pérez, J.I. (2002). Efectos de la participación en el programa de arte Ikertze sobre la creatividad verbal y gráfica. Anales de psicología, 18(1), 95-110. Recuperado de: http://revistas.um.es/analesps/article/ viewFile/28631/27721.

García, J. M. (1989). Una experiencia didáctica con invidentes en el Museo de Cádiz. Cuadernos del Sureste, (1), 46-47.

García, P. (2008). La construcción sinestésica como obra de Arte. Recrearte Revista Internacional de Creatividad aplicada del grupo IACAT Compostela. 8, 1-13.
Garrido, J. (1988). Cómo programar en educación especial. Madrid: Escuela Española.

González, F. y Mitjáns, A. (1999). La Personalidad, su Educación y Desarrollo. La Habana. Cuba: Editorial Pueblo y Educación.

Grunwald, M. (Ed.). (2008). Human haptic perception: Basics and applications. Birkhäuser: Springer Science \& Business Media.

Guilford, J. (1991). Creatividad y Educación. Barcelona: Paidós.

Giúdice, M. (2013). Educación, discapacidad y el desarrollo de la creatividad. Journal for Educators, Teachers and Trainers, 5(1), pp. 90-102.

Halder, S, \& Datta, P. (2012). An exploration into self concept: A comparative analysus between the adolescents who are sighted and blind in India. British Journal of Visual Impairment, 30 (1), 31-41.

Halpin, G., Halpin, G., \& Torrance, E. P. (1973). Effects of blindness on creative thinking abilities of children. Developmental Psychology, 9(2), 268.

Heredia, M. (2009). Artes Plásticas: La comunicación de la experiencia artística en las personas con ceguera. (Tesis inédita de doctorado). Instituto Universitario Nacional del Arte I.U.N.A. pp. 1-64. Recuperado de http://www.arteuna.com/ talleres/tesis/Tesis-Maria Cristina HEREDIA BASAIL.pdf

Hertz, P. (2006). Synesthetic Art- An Imaginary Number?. Leonardo, 32 (5), pp. 399-404 doi: 10.1162/002409499553460.

Hodge, S. \& Eccles, F. (2013). Loneliness, social isolation and sight loss: a literature review conducted for Thomas Pocklington Trust. pp. 1-44. Lancaster University. Recuperado de http:// eprints.lancs.ac.uk/68597/1/loneliness social isolation and sight loss final report dec 13. pdf.

Holmes, E., Hughes, B. \& Jansson, G. (1998). "Haptic perception of texture gradients" Perception 27(8) 993-1008.

Houtz, J.C. \& Phillips, R.H. (1976). Open-Ended Problem-Solving and Creativity ofDisadvantaged Children. The Journal of Creative Behavior, (3). 223. 
Jansson, G. (1988). What are the problems with pictures for the blind, and what can be done to solve them?, In: MagnCe, C. W. M. Vlaskamp, F. J. M. Soede M. and Butcher G. (Eds) Man-Machine Interfaces, Graphics and Pructical Applications. London:Royal National Institute for the Blind.

Jiménez, J., Artiles, C., Rodríguez, C. y García, E. (2007). Adaptación y Baremación del Test de Pensamiento creativo de Torrance: Expresión Figurada. Educación Primaria y Secundaria. Canarias: Consejería de Educación, Cultura y Deportes del gobierno de Canarias.

Khatena, J. (1976). Creative Imagination Imagery: Where is it going?. The Journal of Creative Behavior, 10(3).189-192.

Kennedy, J. M. (1980). Blind people recognizing and making haptic pictures, en Hagen M. A. (de.), The perception of picture, 2, New York: Academic Press.

Kennedy, J. M., \& Domander, R. (1986). Blind people depicting states and events in metaphoric line drawings. Metaphor and Symbol, 1(2), 109-126.

Kirst, A. C. (2010). As aprendizagens do público com deficiência visual: uma experiência de diálogo com a arte contemporânea. Dissertação de Mestrado: Florianópolis: Udesc.

Layden, T., Söffing, C. \& Schmidtke, K. (2015). Comparing the Shape of Sounds: An artistic investigation. $V$ International Conference on Synesteshia, Science and Art, Alcalá la Real, 16-19 ${ }^{\text {th }}$ May. Spain. Recuperado de https://www. researchgate.net/profile/Christine Soeffing/ publication/299657988 Comparing the Shape of Sounds An artistic investigation/

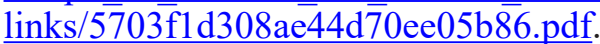

Lobato, S., Martínez, M. y Molinos, I. (2003). El desarrollo de habilidades en las personas con necesidades educativas especiales a través de la expresión plástica. EA Escuela Abierta: Revista de Investigación Educativa. $\mathrm{N}^{\mathrm{o}}$ 6, 47-70.

Lorenzo, J. (2004). La educación artística del deficiente visual. Análisis específico del lenguaje plástico. (Tesis inédita de doctorado). Universidad de La Laguna.

López, O. (2008). Enseñar creatividad. El espacio educativo. Cuad. Fac. Humanid. Cienc. Soc. 12, 49.
López-Torrijo, M. (2009). La Inclusión educativa de alumnos con discapacidades graves y permanentes en la Unión Europea Educational inclusion of students with severe and permanent disabilities in the European Union. RELIEVE. Revista electrónica de investigación y evaluación educativa, 15(1), 1-20.

Lowenfeld, V., \& Brittain, W. L. (1980). Desarrollo de la capacidad creadora. ( $2^{\circ}$ Ed.). Buenos Aires: Kapelusz.

Lozano, J., Cerezo, M. y Alcaraz, S. (2015). Plan de Atención a la Diversidad. Madrid: Alianza.

Llanos, M. (2006). Celebrando la luz de la ceguera. Experiencia pionera en biodanza con adultos ciegos. Lima, Perú. Recuperado de http:// www.monografias.com/trabajos-pdf5/biodanza-y-discapacidad-celebrando-luz-ceguera/biodanza-y-discapacidad-celebrando-luz-ceguera. shtml.

Limiñana, R., Bordoy, M., Juste, G. \& Corbalán, F. (2010). Creativity, intelectual abilities and response styles: Implications for academic performance in the secondary school. Anales de Psicología/Annals of Psychology, 26(2), 212-219.

Malchiodi, A. (2003). Hand book of art Therapy. New York: the Guilford press.

Marín, R. (1995). La Creatividad: diagnóstico, evaluación e investigación. Madrid: UNED.

Marina, J. y Marina, E. (2013). El aprendizaje de la creatividad. Barcelona. Ariel.

Martínez Abellán, R. (2005a). Deficiencia visual, creatividad, expresión y terapias artísticas (I) . Polibea, (74), pp. 15-26.

Martínez Abellán, R. (2005b). Deficiencia visual, creatividad, expresión y terapias artísticas (II). Polibea, (75), pp.29-43.

MEC (2012). Ministerio de Educación. Educación Inclusiva: Personas con Discapacidad Visual. Módulo 3: Desarrollo Evolutivo.

Mendoza, Y. (2001). El maestro creativo. Algunas reflexiones en torno a su existencia. Educere, 5(15). Recuperado de: http:// www.saber.ula.ve/handle/123456789/19641. 
Millar, S. (1994). Understanding and representing space: Theory and evidence from studies with blind and sighted children. Oxford UK : Oxford University Press.

Mitjáns, A. (1996). Creatividad en la educación especial. Siglo XXI. Perspectivas de la educación desde América Latina, 2(5), 28.

Mitjáns Martínez, A. (1997) Creatividad y educación especial. En Betancourt, J. Morejón, A. Mitjáns Martínez, S. de la Torre, P. Solís. Cámara Resendiz Pensar y Crear. Educar para el cambio. (71-88) La Habana. Académica.

Mishra, V. \& Singh, A. (2012). A comparative study of self-concept and self-confidence of sighted and visually impaired children. Internacional Journal of Multidisciplinary Management studies, 2 (2), 148-157.

Monreal, C. (2000). Qué es la creatividad. Madrid: Biblioteca Nueva. 57-62.

Moreno, M., Huijbregt, L. y Ramírez, A. (2012). Conocimiento estético y percepción háptica en la experiencia del arte como motor en las relaciones humanas. Red Visual, 17 15-22.Recuperado de http://www.redvisual.net/images/PDF/17/ redvisual17 02 moreno-huijbergt-ramirez.pdf.

Muñoz, A. (2015). Manual de creatividad (Textos Docentes). Madrid: Fundación Universitaria San Pablo CEU, 1.

Novaes, M.H. (1979). Psicología de la aptitud creadora. Buenos Aires: Kapelusz, Argentina.

Nuñez, M. (2001). La deficiencia visual. En Memorias del III Congreso "La atención a la diversidad en el sistema educativo", Universidad de Salamanca, Instituto Universitario de Integración en la Comunidad. ONCE. Recuperado de https://campus.usal.es/ inico/actividades/ actasuruguay2001/10.pdf.

Penagos, J. C. (1995). Efectos de los procesos de comunicación profesor alumno y de la práctica de técnicas de autoconciencia, en niños, sobre el aprendizaje; una aproximación epistemológica experimental hacia el constructivismo (Doctoral dissertation, Tesis de maestría no publicada. Puebla. Cholula: Universidad de las Américas).

Penagos, J. (1997). El origen de la creatividad. Calidad y Excelencia, 2(13), 4-8.

Penagos, J. y Aluni, R. (2000). Creatividad, una aproximación. Revista Psicología (Edición Especial), 1-8.
Peña, N. (2014). Otras visualidades: crear y enseñar fotografia desde la percepción invidente. (Tesis inédita de doctorado). Universidad Complutense de Madrid. 29-110.

Pérez-Pereira, M. \& Conti-Ramsden, G. (2013). Language development and social interaction in blind children. Psychology Press.

Pitman, D. J. (1965). The musical ability of blind children. Review of Psychology of Music. Vol. 2(2), 19-28.

Pinquart, M. \& Pfeiffer, J. P. (2012). Body image in adolescents with and without visual impairment. British Journal of Visual Impairment, 30(3), 122-131.

Pujolás, P. (2010). No es inclusión todo lo que se dice que lo es. Aula de innovación educativa, $n .^{\circ} 191$, $38-41$.

Revesz, G. (1950). The psychology and art of the blind. London: Longmans Green.

Riley, S. (2001). Art therapy with adolescents. The Western Journal of Medicine, 175 (1), 54 Recuperado de: http://www.ewjm.com/cgi/ content/full/175/1/54.

Rocío, G. (2011). La cultura NO es para todos. De cómo abordar desde una perspectiva educativa y social la atención a la diversidad utilizando como vehículo el Arte. Temas para la Educación, revista digital para profesionales de la enseñanza, $\mathrm{N}^{\mathrm{o}}$ 14. pp 1-7.

Rodríguez, J.A. (2002). La mente de los creadores. Un estudio de los procesos creativos desde la neurociencia y la psicología. Madrid: Biblioteca Nueva.

Rodríguez, A. (2003). Integración escolar de alumnos con deficiencia visual en España: Algunas sugerencias espaciales y contribuciones tecnológicas y tiflotecnológicas. Estudios pedagógicos (Valdivia), (29), 143-153.

Rosa, B. (2008). Estrategias docentes en el desarrollo de la creatividad escolar. REDHECS: Revista electrónica de Humanidades, Educación y Comunicación Social, 3, (5), pp. 65-76.

Romo, M. (1997). Psicología de la creatividad. Barcelona: Paidós. 
Romo, M. E., López, D. y López, I. (2006). ¿Eres visual, auditivo o kinestésico?: Estilos de aprendizaje desde el modelo de la Programación Neurolingüística (PNL). Universidad de Chile. Revista Iberoamericana de Educación, 38(2), 6. Recuperado de: http://rieoei.org/1274.htm.

Romo, M. (2012). Algunas investigaciones sobre el impacto de la creatividad en el ámbito educativo. Good Morning Creativity, 123.

Romero, S. (2013). Creativity in education, its development from a pedagogical perspective. Journal of sport and health research, 5(2), 221-228.

Rubin, J.A. (2009). Introduction to Art Therapy. Londres: Roultledge.

Rubin, J.A (2011). Child art therapy. 25 th Anniversary Edition. New York: John Wiley \& Sons. 90-110.

Runco, M. A. (2014). Creativity: Theories and themes: Research, development, and practice. Elsevier.

Ruiz, M. (2004). Discapacidad y sociedad: un programa educativo en el museo dirigido a personas con discapacidad visual. Revista de Enseñanza Universitaria, (23), 47-62.

Salas, J. (2015). Sinestesia y arte. Hacia la autoinvestigación creativa. (Tesis doctoral). UGR. Recuperado de: https://hera.ugr.es/ tesisugr/25934922.pdf.

Sternberg, R. y Lubart, T. (1997). La creatividad en una cultura conformista. Un desafío a las masas. Barcelona: Paidós.

Tapia, I. C. (2007). Psicología de la ceguera. Recuperado http://www.integrando.org.ar/ datosdeinteres/it psicologia ceguera.htm.

Tisdall, W. J., Blackhurst, A. E., \& Marks, C. H. (1971). Divergent thinking in blind children. Journal of educational psychology, 62(6), 468.

Tilley, P. (1991).El arte en la educación especial. Barcelona: Ceac.

Toro, J. (2008). La creatividad del "co-razón". Creatividad y sociedad: revista de la Asociación para la Creatividad, (12), 6-20.

Torretti, R. (2008). Pseudo-Longino, De lo sublime. Traducción de Eduardo Molina C. y Pablo Oyarzun R. Noticia Preliminar, Notas e índices de Pablo Oyarzun R. Revista de filosofia, 64, 264-265.
Uno, T., Gargiulo, R. M., Sears, J. D., Mauter, M \& Rowe, J. (1976). Creative Behavior of Trainable and Educable Mentally Retarded Adolescents. The Journal of Creative Behavior, 10 (3). 221.

Vanlierde, A. \& Wanet-Defalque, M. (2005). The role of visual experience in mental imagery. Journal of Visual Impairment \& Blindness (JVIB), 99 (03) $165-178$.

Warren, D. (1994). Blindness and children: An individual differences approach. Cambridge: University Press.

Witkin, H. A., Birnbaum, J., Lomonaco, S., Lehr, S., \& Herman, J. L. (1968). Cognitive patterning in congenitally blind children. Child Development, 39 , 767-786. 